\title{
Multiplicity of Periodic Solutions for Third-Order Nonlinear Differential Equations
}

\author{
Xuxin Yang, ${ }^{1}$ Weibing Wang, ${ }^{2}$ and Dingyang $\mathbf{L v}^{1}$ \\ ${ }^{1}$ Department of Mathematics, Hunan First Normal University, Changsha, Hunan 410205, China \\ ${ }^{2}$ Department of Mathematics, Hunan University of Science and Technology, Xiangtan, Hunan 411201, China \\ Correspondence should be addressed to Xuxin Yang; yangxx2002@sohu.com
}

Received 29 November 2014; Revised 16 March 2015; Accepted 20 March 2015

Academic Editor: Alexander Klimenko

Copyright (c) 2015 Xuxin Yang et al. This is an open access article distributed under the Creative Commons Attribution License, which permits unrestricted use, distribution, and reproduction in any medium, provided the original work is properly cited.

We study the existence of periodic solutions for third-order nonlinear differential equations. The method of proof relies on Schauder's fixed point theorem applied in a novel way, where the original equation is transformed into second-order integrodifferential equation through a linear integral operator. Finally, examples are presented to illustrate applications of the main results.

\section{Introduction}

Questions on the existence and the multiplicity of periodic solutions are important topics in qualitative analysis of differential equations. Much work related to periodic solutions for second-order differential equations has been done by using various theorems and methods of nonlinear functional analysis; see [1-10] and references therein. In this paper, we investigate existence of periodic solutions of the following differential equation:

$$
u^{\prime \prime \prime}(t)=g(u(t))-f(t, u(t)),
$$

where $g(u): R \rightarrow R, f(t, u)$ is $\omega$-periodic in $t$, and $\omega>$ 0 . Third-order differential equations of the above type arise, for example, in various fields of agriculture, biology, economics, and physics [11-14]. Questions related to this class of differential equations have recently attracted considerable attention from the researcher community; see, for example, [15-19].

A naive idea in study of higher-order (in particular thirdorder) differential equations is to translate the equation into a first-order system of differential equations by defining $x_{1}=$ $x, x_{2}=x^{\prime}, x_{3}=x^{\prime \prime}, \ldots$. This method works well, if the task is to show the existence of periodic solutions. However, it does not obviously lead to existence proofs for positive periodic solutions, since the condition $x=x_{1} \geq 0$ of positivity for the higher-order equation is different from the natural positivity condition $\left(x_{1}, x_{2}, \ldots \geq 0\right)$ for the corresponding system.

Another frequently used approach is to transform the third-order equation into a corresponding integral equation and to establish the existence of positive periodic solutions based on a fixed point theorem in cones. In order to follow this path, one needs an explicit representation of Green's function for corresponding ordinary equation; see [20, 21]. In [20], Agarwal gave the explicit Green function for the $n$ thorder and $2 m$ th-order differential equations. Futhermore, Anderson has studied Green's function for a third-order boundary value problem in [21].

It should be noted that (1) includes many important models. For example, it arises in many fields of science and technology, such as physics, mechanics, and engineering. We refer the reader to [22-27] for recent results on such models.

The main purpose of this paper is to show the existence of periodic solutions of (1) by means of Schauder's fixed point theorem. The method of proof used in this paper is based on a simple but novel idea, and it consists of two steps as follows.

(1) The first step is to transform the original equation into a second-order integrodifferential equation through a linear integral operator. 
(2) The second step is to apply Schauder's fixed point theorem.

After the above steps, the existence of a single periodic solution for (1) has been established under suitable behavior of functions $g$ and $f$ on some closed set. In addition, some information on the location of a periodic solution is obtained, leading to results on the multiplicity of solutions. To our belief, neither this method nor similar results can be found in the literature.

The paper is organized as follows. In Section 2 we introduce a lemma, which is crucial in proving the main results. Section 3 is devoted to the existence results on solutions of (1). In Section 4 we give some examples to illustrate potential applications of the main results.

\section{Preliminaries}

Let $X=\{u \in C(R, R): u(t+\omega)=u(t)$ for all $t \in R\}$ with the norm $\|u\|=\max _{t \in[0, \omega]}|u(t)|$; then $X$ is a Banach space.

Let $p>0, h_{1}, h_{2} \in X$, and consider the following two differential equations:

$$
\begin{aligned}
& u^{\prime \prime}-p u^{\prime}+p^{2} u=h_{1}(t), \\
& u^{\prime \prime}+p u^{\prime}+p^{2} u=h_{2}(t) .
\end{aligned}
$$

Lemma 1. Assume that $0<p \omega<2 \pi / \sqrt{3}$. Then (2) has a unique $\omega$-periodic solution $\bar{u}$ satisfying

$$
\bar{u}(t)=\int_{0}^{\omega} G(t, s) h_{1}(s) d s, \quad t \in[0, \omega],
$$

and (3) has a unique $\omega$-periodic solution $\widehat{u}$ satisfying

$$
\widehat{u}(t)=\int_{0}^{\omega} H(t, s) h_{2}(s) d s, \quad t \in[0, \omega]
$$

where

$$
\begin{aligned}
& G(t, s) \\
& =\frac{1}{A} \\
& .\left\{\begin{array}{l}
e^{(p / 2)(\omega+t-s)} \\
\cdot\left[\sin \frac{\sqrt{3}}{2} p(\omega+t-s)+e^{(p / 2) \omega} \sin \frac{\sqrt{3}}{2} p(s-t)\right], \\
e^{(p / 2)(\omega+t-s)} \\
\cdot\left[\sin \frac{\sqrt{3}}{2} p(\omega+s-t)+e^{-(p / 2) \omega} \sin \frac{\sqrt{3}}{2} p(t-s)\right], \\
t \geq s,
\end{array}\right.
\end{aligned}
$$

$$
\begin{aligned}
& H(t, s) \\
& =\frac{1}{B} \\
& \left\{\begin{array}{l}
e^{-(p / 2)(\omega+t-s)} \\
\cdot\left[\sin \frac{\sqrt{3}}{2} p(\omega+t-s)+e^{-(p / 2) \omega} \sin \frac{\sqrt{3}}{2} p(s-t)\right] \\
0 \leq t \leq s, \\
e^{-(p / 2)(\omega+t-s)} \\
\cdot\left[\sin \frac{\sqrt{3}}{2} p(\omega+s-t)+e^{(p / 2) \omega} \sin \frac{\sqrt{3}}{2} p(t-s)\right] \\
\quad t \geq s, \\
A=\sqrt{3}\left(\frac{1+e^{p \omega}}{2}-e^{(p / 2) \omega} \cos \frac{\sqrt{3}}{2} p \omega\right) p>0, \\
B=\sqrt{3}\left(\frac{1+e^{-p \omega}}{2}-e^{-(p / 2) \omega} \cos \frac{\sqrt{3}}{2} p \omega\right) p>0 .
\end{array}\right.
\end{aligned}
$$

Proof. We only consider (2). Let $u_{1}, u_{2}$ be two $\omega$-periodic solutions of (2). Then, $u^{*}=u_{1}-u_{2}$ is $\omega$-periodic solution of the differential equation

$$
u^{\prime \prime}-p u^{\prime}+p^{2} u=0 .
$$

Note that $u^{*}$ can be written as

$$
u^{*}=c_{1} e^{(p / 2) t} \cos \frac{\sqrt{3}}{2} p t+c_{2} e^{(p / 2) t} \sin \frac{\sqrt{3}}{2} p t .
$$

From $u^{*(i)}(0)=u^{*(i)}(\omega), i=0,1$, we obtain $c_{1}=c_{2}=0$. Thus $u_{1}=u_{2}$.

Next, we show that

$$
\begin{gathered}
\bar{u}^{\prime \prime}-p \bar{u}^{\prime}+p^{2} \bar{u}=h_{1}(t), \quad t \in[0, \omega], \\
\bar{u}^{(i)}(0)=\bar{u}^{(i)}(\omega), \quad i=0,1 .
\end{gathered}
$$

By direct computation, we get

$$
\begin{aligned}
\bar{u}^{\prime}(t) & \frac{p}{2 A} \\
& \cdot \int_{0}^{t} e^{(p / 2)(\omega+t-s)} h_{1}(s) \\
& \cdot\left(\sin \frac{\sqrt{3}}{2} p(\omega+s-t)+e^{-p \omega / 2} \sin \frac{\sqrt{3} p}{2}(t-s)\right) d s \\
+ & \frac{\sqrt{3} p}{2 A} \\
& \cdot \int_{0}^{t} e^{(p / 2)(\omega+t-s)} h_{1}(s) \\
& \cdot\left(-\cos \frac{\sqrt{3}}{2} p(\omega+s-t)+e^{-p \omega / 2} \cos \frac{\sqrt{3} p}{2}(t-s)\right) d s
\end{aligned}
$$




$$
\begin{aligned}
& +\frac{p}{2 A} \\
& \cdot \int_{t}^{\omega} e^{(p / 2)(\omega+t-s)} h_{1}(s) \\
& \cdot\left(\sin \frac{\sqrt{3}}{2} p(\omega+t-s)+e^{p \omega / 2} \sin \frac{\sqrt{3} p}{2}(s-t)\right) d s \\
& +\frac{\sqrt{3} p}{2 A} \\
& \cdot \int_{t}^{\omega} e^{(p / 2)(\omega+t-s)} h_{1}(s) \\
& \cdot\left(\cos \frac{\sqrt{3}}{2} p(\omega+t-s)-e^{p \omega / 2} \cos \frac{\sqrt{3} p}{2}(s-t)\right) d s, \\
& \bar{u}^{\prime \prime}(t) \\
& =-\frac{p^{2}}{2 A} \\
& \cdot \int_{0}^{t} e^{(p / 2)(\omega+t-s)} h_{1}(s) \\
& \cdot\left(\sin \frac{\sqrt{3}}{2} p(\omega+s-t)+e^{-p \omega / 2} \sin \frac{\sqrt{3} p}{2}(t-s)\right) d s \\
& +\frac{\sqrt{3} p^{2}}{2 A} \\
& \int_{0}^{t} e^{(p / 2)(\omega+t-s)} h_{1}(s) \\
& \cdot\left(-\cos \frac{\sqrt{3}}{2} p(\omega+s-t)+e^{-p \omega / 2} \cos \frac{\sqrt{3} p}{2}(t-s)\right) d s \\
& -\frac{p^{2}}{2 A} \\
& \cdot \int_{t}^{\omega} e^{(p / 2)(\omega+t-s)} h_{1}(s) \\
& \cdot\left(\sin \frac{\sqrt{3}}{2} p(\omega+t-s)+e^{p \omega / 2} \sin \frac{\sqrt{3} p}{2}(s-t)\right) d s \\
& +\frac{\sqrt{3} p^{2}}{2 A} \\
& \cdot \int_{t}^{\omega} e^{(p / 2)(\omega+t-s)} h_{1}(s) \\
& \cdot\left(\cos \frac{\sqrt{3}}{2} p(\omega+t-s)-e^{p \omega / 2} \cos \frac{\sqrt{3} p}{2}(s-t)\right) d s .
\end{aligned}
$$

Hence, we have

$$
\bar{u}^{\prime \prime}-p \bar{u}^{\prime}+p^{2} \bar{u}=h_{1}(t)
$$

Moreover, it is easy to check that $\bar{u}^{(i)}(0)=\bar{u}^{(i)}(\omega), \quad i=0,1$.
Remark 2. If $0<p \omega<2 \pi / \sqrt{3}$, then

$$
G(t, s)>0, \quad H(t, s)>0, \quad t, s \in[0, \omega] .
$$

Remark 3. Consider

$$
\int_{0}^{\omega} G(t, s) d s=\frac{1}{p^{2}}, \quad \int_{0}^{\omega} H(t, s) d s=\frac{1}{p^{2}} .
$$

Define two operators $T, S: C^{0}(X) \rightarrow C^{2}(X)$ by

$$
\begin{aligned}
& T: h_{1} \longrightarrow \bar{u}(t)=T h_{1}, \\
& S: h_{2} \longrightarrow \widehat{u}(t)=S h_{2} .
\end{aligned}
$$

From Lemma 1, one can easily check that $T, S$ are compact, increasing operators for $0<p \omega<2 \pi / \sqrt{3}$.

Remark 4. If $h \equiv c$ is a constant function, then

$$
T h=\frac{c}{p^{2}}, \quad S h=\frac{c}{p^{2}},
$$

for $0<p \omega<2 \pi / \sqrt{3}$.

Next, we define two operators $J$ and $K$ on $X$ by

$$
\begin{gathered}
(J u)(t)=\int_{t}^{t+\omega} \frac{e^{(s-t) p}}{e^{p \omega}-1} u(s) d s, \quad u \in X, \\
(K u)(t)=\int_{t}^{t+\omega} \frac{e^{(t+\omega-s) p}}{e^{p \omega}-1} u(s) d s, \quad u \in X,
\end{gathered}
$$

where $p>0$ is a constant. For any $u \in X, J u \in X \cap C^{1}(R)$ and $K u \in X \cap C^{1}(R)$.

Lemma 5. If $u \in X \cap C^{2}(R)$ satisfies the differential equation

$$
u^{\prime \prime}-p u^{\prime}+p^{2} u=g(J u)-f(t, J u)+p^{3}(J u),
$$

then $J u$ is a $\omega$-periodic solution of (1).

If $u \in X \cap C^{2}(R)$ satisfies the differential equation

$$
-u^{\prime \prime}-p u^{\prime}-p^{2} u=g(K u)-f(t, K u)-p^{3}(K u),
$$

then $\mathrm{Ku}$ is a w-periodic solution of (1).

Proof. Note that

$$
\begin{aligned}
& (J u)(t+\omega) \\
& =\int_{t+\omega}^{t+2 \omega} \frac{e^{(s-t-\omega) p}}{e^{p \omega}-1} u(s) d s \\
& =\int_{t}^{t+\omega} \frac{e^{(r-t) p}}{e^{p \omega}-1} u(r+\omega) d r \\
& =(J u)(t), \quad u \in X,
\end{aligned}
$$

$$
(J u)^{\prime}(t)=-p(J u)(t)+u(t) .
$$

If $u \in X \cap C^{1}(R), J u \in X \cap C^{2}(R)$ and

$$
\begin{aligned}
(J u)^{\prime \prime}(t) & =-p(J u)^{\prime}(t)+u^{\prime}(t) \\
& =p^{2}(J u)(t)-p u(t)+u^{\prime}(t) .
\end{aligned}
$$


If $u \in X \cap C^{2}(R), J u \in X \cap C^{3}(R)$ and

$$
\begin{aligned}
(J u)^{\prime \prime \prime}(t) & =p^{2}(-p(J u)(t)+u(t))-p u^{\prime}(t)+u^{\prime \prime}(t) \\
& =-p^{3}(J u)(t)+p^{2} u(t)-p u^{\prime}(t)+u^{\prime \prime}(t) .
\end{aligned}
$$

Hence

$$
(J u)^{\prime \prime \prime}(t)+p^{3}(J u)(t)=u^{\prime \prime}(t)-p u^{\prime}(t)+p^{2} u(t) .
$$

If

$$
\begin{aligned}
u^{\prime \prime}(t) & -p u^{\prime}(t)+p^{2} u(t) \\
= & h((J u)(t)):=g((J u)(t))-f(t,(J u)(t)) \\
& +p^{3}(J u)(t),
\end{aligned}
$$

then

$$
\begin{aligned}
& (J u)^{\prime \prime \prime}(t)+p^{3}(J u)(t) \\
& \quad=g((J u)(t))-f(t,(J u)(t))+p^{3}(J u)(t) .
\end{aligned}
$$

If $u \in X \cap C^{2}(R)$ satisfies (17), we have

$$
(J u)^{\prime \prime \prime}(t)=g((J u)(t))-f(t,(J u)(t)) .
$$

Hence, $J u$ is a $\omega$-periodic solution of (1).

On the other hand, we have

$$
(K u)^{\prime}(t)=p(K u)(t)-u(t) .
$$

If $u \in X \cap C^{1}(R)$, then $K u \in X \cap C^{2}(R)$ and

$$
\begin{aligned}
(K u)^{\prime \prime}(t) & =p(K u)^{\prime}(t)-u^{\prime}(t) \\
& =p^{2}(K u)(t)-p u(t)-u^{\prime}(t) .
\end{aligned}
$$

From the above, we see that if $u \in X \cap C^{2}(R)$, then $K u \in$ $X \cap C^{3}(R)$ and

$$
\begin{aligned}
(K u)^{\prime \prime \prime}(t) & =p^{2}(p(K u)(t)-u(t))-p u^{\prime}(t)-u^{\prime \prime}(t) \\
& =p^{3}(K u)(t)-p^{2} u(t)-p u^{\prime}(t)-u^{\prime \prime}(t) .
\end{aligned}
$$

We have

$$
(K u)^{\prime \prime \prime}(t)-p^{3}(K u)(t)=-p^{2} u(t)-p u^{\prime}(t)-u^{\prime \prime}(t) .
$$

If

$$
\begin{aligned}
- & \left(u^{\prime \prime}(t)+p u^{\prime}(t)+p^{2} u(t)\right) \\
& =\bar{h}((K u)(t)) \\
& :=g((K u)(t))-f(t,(K u)(t))-p^{3}(K u)(t),
\end{aligned}
$$

then

$$
\begin{aligned}
& (K u)^{\prime \prime \prime}(t)-p^{3}(K u)(t) \\
& \quad=g((K u)(t))-f(t,(K u)(t))-p^{3}(K u)(t) .
\end{aligned}
$$

If $u \in X \cap C^{2}(R)$ satisfies (18), we have

$$
(K u)^{\prime \prime \prime}(t)=g((K u)(t))-f(t,(K u)(t)) .
$$

Hence, $K u$ is a $\omega$-periodic solution of (1).
Lemma 6 (see [28]). Let $X$ be a Banach space so that $D \subset X$ is closed and convex. Assume that $T: D \rightarrow D$ is a completely continuous operator. Then $T$ has a fixed point in $D$.

\section{Main Results}

The following theorems are the main results of this paper.

Theorem 7. Assume that there exist constants $m<M$ such that

$\left(H_{1}\right) g \in C^{1}[m, M]$ and $g^{\prime}(u)<(2 \pi / \sqrt{3} \omega)^{3}$ in $u \in[m, M]$;

$\left(H_{2}\right) f(t, u) \in C(R \times[m, M])$ and

$$
g(m) \leq f(t, u) \leq g(M)
$$

for any $(t, u) \in R \times[m, M]$.

Then (1) has at least one $\omega$-periodic solution $u$ with $m \leq$ $u \leq M$.

Proof. From $\left(H_{1}\right)$, we obtain that there exists a constant $p \in$ $(0,2 \pi / \sqrt{3} \omega)$ with $g^{\prime}(u)<p^{3}$ in $u \in[m, M]$. Consider the differential equation

$$
u^{\prime \prime}+p u^{\prime}+p^{2} u=f(t, K u)+p^{3}(K u)-g(K u) .
$$

From Lemma 1 we see that if $u$ is a solution of (34), then $u$ satisfies

$$
u=(S \circ W) u,
$$

where $S \circ W$ is composition of $S$ defined by $(S \circ W) u=S(W u)$ and the operator $W$ is defined by

$$
(W u)(t)=f(t, K u)+p^{3}(K u)-g(K u) .
$$

Set $\Omega=\{u \in X: p m \leq u(t) \leq p M, t \in R\}$. For all $u \in \Omega$, we have $m \leq K u \leq M$. Put $G(u)=p^{3} u-g(u)$; then $G^{\prime}(u)=p^{3}-g^{\prime}(u)>0$ for $u \in[m, M]$. Thus

$$
p^{3} m-g(m) \leq G(u) \leq p^{3} M-g(M)
$$

for any $u \in[m, M]$. Hence, for all $u \in \Omega$,

$$
\begin{aligned}
p^{3} m-g(m) & \leq G(K u)=p^{3}(K u)(t)-g((K u)(t)) \\
& \leq p^{3} M-g(M) .
\end{aligned}
$$

Using $\left(H_{2}\right)$, we obtain that, for all $u \in \Omega$,

$$
\begin{aligned}
(W u)(t) & =p^{3}(K u)-g(K u)+f(t, K u) \\
& \leq p^{3} M-g(M)+f(t, K u) \leq p^{3} M, \\
(W u)(t) & =p^{3} K u(t)-g(K u)+f(t, K u) \\
& \geq p^{3} m-g(m)+f(t, K u) \geq p^{3} m .
\end{aligned}
$$

Since $S$ is an increasing operator, we obtain that, for $u \in \Omega$,

$$
S\left(p^{3} m\right) \leq(S \circ W) u \leq S\left(p^{3} M\right) .
$$


By Remark 4, we have

$$
p m \leq(S \circ W) u \leq p M
$$

for $u \in \Omega$; that is, $(S \circ W)(\Omega) \subset \Omega$.

Also, from the facts that $S$ is completely continuous and $W$ is continuous it follows that $S \circ W: \Omega \rightarrow \Omega$ is a continuous and compact map. By Lemma $6, S \circ W$ has at least one fixed point $u$ in $\Omega$ and $m \leq K u \leq M$ is periodic solution of (1). The proof is complete.

Theorem 8. Assume that there exist constants $m<M$ such that

$$
\begin{aligned}
& \left(H_{3}\right) g \in C^{1}[m, M] \text { and } g^{\prime}(u) \geq-(2 \pi / \sqrt{3} \omega)^{3} \text { in } u \in \\
& {[m, M]} \\
& \left(H_{4}\right) f(t, u) \in C(R \times[m, M]) \text { and }
\end{aligned}
$$

$$
g(M) \leq f(t, u) \leq g(m)
$$

for any $(t, u) \in R \times[m, M]$.

Then (1) has at least one $\omega$-periodic solution $u$ with $m \leq$ $u \leq M$.

Proof. From $\left(\mathrm{H}_{3}\right)$, we obtain that there exists a constant $p \epsilon$ $(0,2 \pi / \sqrt{3} \omega)$ with $g^{\prime}(u) \geq-p^{3}$ in $u \in[m, M]$. Consider the differential equation

$$
u^{\prime \prime}-p u^{\prime}+p^{2} u=g(J u)-f(t, J u)+p^{3}(J u) .
$$

From Lemma 1 , if $u$ is a solution of (43), $u$ satisfies

$$
u=(T \circ V) u,
$$

where $T \circ V$ is composition of $T$ and $V$ defined as $(T \circ V) u=$ $T(V u)$, and the operator $V$ is defined as

$$
(V u)(t)=g(J u)+p^{3}(J u)-f(t, J u) .
$$

Set $\Omega=\{u \in X: p m \leq u(t) \leq p M, t \in R\}$, where $p \in$ $(0,2 \pi / \sqrt{3} \omega)$ with $g^{\prime}(u) \geq-p^{3}$ in $[m, M]$. We set $I(u)=u+$ $\left(1 / p^{3}\right) g(u)$; then $I^{\prime}(u)=1+\left(1 / p^{3}\right) g^{\prime}(u) \geq 0$; we have

$$
m+\frac{1}{p^{3}} g(m) \leq I(u) \leq M+\frac{1}{p^{3}} g(M),
$$

for any $u \in[m, M]$. Hence, for all $u \in \Omega$, we have

$$
m+\frac{1}{p^{3}} g(m) \leq I(J u) \leq M+\frac{1}{p^{3}} g(M) .
$$

Using $\left(H_{4}\right)$, we obtain that, for all $u \in \Omega$,

$$
\begin{aligned}
(V u)(t) & =p^{3}(J u)+g(J u)-f(t, J u) \\
& \leq p^{3} M+g(M)-f(t, J u) \leq p^{3} M, \\
(V u)(t) & =p^{3}(J u)+g(J u)-f(t, J u) \\
& \geq p^{3} m+g(m)-f(t, J u) \geq p^{3} m .
\end{aligned}
$$

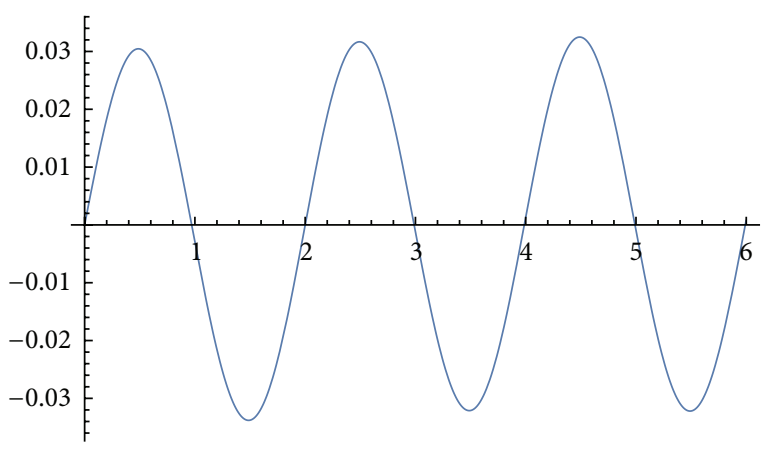

FIgURE 1: A numerical approximation to a 2-periodic solution to (51). The approximation is obtained by using the built-in NDsolve function of Mathematica 10.

Since $T$ is an increasing operator, we obtain that, for $u \in \Omega$,

$$
T\left(p^{3} m\right) \leq(T \circ V) u \leq T\left(p^{3} M\right) .
$$

By Remark 4, we have

$$
p m \leq(T \circ V) u \leq p M
$$

for $u \in \Omega$; that is, $(T \circ V)(\Omega) \subset \Omega$.

Also, from the facts that $T$ is completely continuous and $V$ is continuous, it follows that $T \circ V: \Omega \rightarrow \Omega$ is a continuous and compact map. By Lemma 6, $T \circ V$ has at least one fixed point in $\Omega$. The proof is complete.

\section{Some Examples}

In this section, two examples are provided to highlight potential applications of the results obtained in the previous section.

Example 1. Consider the differential equation

$$
x^{\prime \prime \prime}=\sin x-\cos \left(\pi t+x^{2}\right) .
$$

Note that here

$$
\begin{gathered}
g(x)=\sin x, \quad \omega=2, \quad f(t, x)=\cos \left(\pi t+x^{2}\right), \\
g^{\prime}(x) \leq 1<\left(\frac{2 \pi}{\sqrt{3} \omega}\right)^{3}, \quad \forall x \in R .
\end{gathered}
$$

Letting $M=2 n \pi+(1 / 2) \pi, m=2 n \pi-\pi / 2, n \in Z$,

$$
g(m)=-1 \leq f(t, x) \leq 1=g(M) .
$$

By Theorem 7, (51) has infinitely many 2-periodic solutions. One such solution is illustrated in Figure 1.

Example 2. Consider the differential equation

$$
x^{\prime \prime \prime}=\frac{1}{2} x(x-1)-\frac{1}{20} e^{x^{2} / 2+\sin 2 t / 2} .
$$




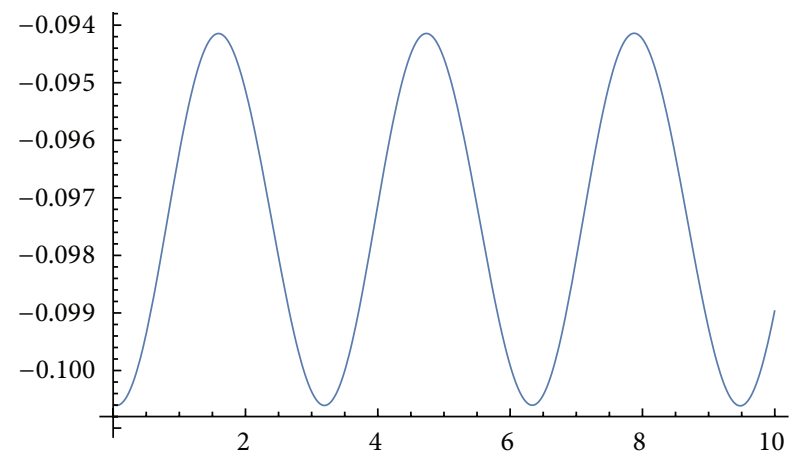

FIgURE 2: A numerical approximation of a $\pi$-periodic solution to (54). The approximation is obtained by using the built-in NDsolve function of Mathematica 10.

We claim that (54) has at least two $\pi$-periodic solutions. Note that here

$$
\begin{gathered}
g(x)=\frac{1}{2} x(x-1), \quad f(t, x)=\frac{1}{20} e^{x^{2} / 2+\sin 2 t / 2}, \\
\omega=\pi .
\end{gathered}
$$

Let $m_{1}=-1 / 2, M_{1}=1 / 2, m_{2}=1$, and $M_{2}=2$. Then

$$
\begin{gathered}
g^{\prime}(x)=x-\frac{1}{2} \geq-1>-\left(\frac{2 \pi}{\sqrt{3} \omega}\right)^{3}, \quad \forall x \in\left[m_{1}, M_{1}\right], \\
g^{\prime}(x)=x-\frac{1}{2} \leq \frac{3}{2}<\left(\frac{2 \pi}{\sqrt{3} \omega}\right)^{3}, \quad \forall x \in\left[m_{2}, M_{2}\right], \\
g\left(M_{1}\right)=-\frac{1}{8}, \quad g\left(m_{1}\right)=\frac{1}{8}, \\
g\left(M_{2}\right)=1, \quad g\left(m_{2}\right)=0, \\
g\left(M_{1}\right)<\frac{1}{20}<f(t, x) \leq \frac{1}{20} e^{5 / 8}<\frac{1}{8}=g\left(m_{1}\right), \\
\forall x \in\left[m_{1}, M_{1}\right], \\
g\left(m_{2}\right)<f(t, x) \leq \frac{1}{20} e^{5 / 2}<1=g\left(M_{2}\right), \\
\forall x \in\left[m_{2}, M_{2}\right] .
\end{gathered}
$$

By Theorems 7 and 8, (54) has at least two $\pi$-periodic solutions $x_{i} \in\left[m_{i}, M_{i}\right], i=1,2$. One such solution is illustrated in Figure 2.

\section{Conflict of Interests}

The authors declare that there is no conflict of interests regarding the publication of this paper.

\section{Acknowledgments}

This work is supported by the NNSF of China (11171085), Hunan Provincial Natural Science Foundation of China
(14JJ7083), and a Key Project supported by Scientific Research Fund of Hunan Provincial Education Department (14A028) and supported by Scientific Research Fund of Hunan Provincial Education Department (14C0253).

\section{References}

[1] C. Bereanu and J. Mawhin, "Multiple periodic solutions of ordinary differential equations with bounded nonlinearities and $\phi$-Laplacian," NoDEA. Nonlinear Differential Equations and Applications, vol. 15, no. 1-2, pp. 159-168, 2008.

[2] W.-S. Cheung and J. Ren, "On the existence of periodic solutions for $p$-Laplacian generalized Liénard equation," Nonlinear Analysis: Theory, Methods \& Applications, vol. 60, no. 1, pp. 65-75, 2005.

[3] C. de Coster and P. Habets, "Upper and lower solutions in the theory of ODE boundary value problems: classical and recent results," in Nonlinear Analysis and Boundary Value Problems for Ordinary Differential Equations, CISM-ICMS, F. Zanolin, Ed., vol. 371, pp. 1-78, Springer, New York, NY, USA, 1996.

[4] A. Fonda and L. Ghirardelli, "Multiple periodic solutions of scalar second order differential equations," Nonlinear Analysis: Theory, Methods \& Applications, vol. 72, no. 11, pp. 4005-4015, 2010.

[5] Z. Guo and J. Yu, "Multiplicity results for periodic solutions to delay differential equations via critical point theory," Journal of Differential Equations, vol. 218, no. 1, pp. 15-35, 2005.

[6] A. C. Lazer, "On Schauder's fixed point theorem and forced second-order nonlinear oscillations," Journal of Mathematical Analysis and Applications, vol. 21, pp. 421-425, 1968.

[7] A. C. Lazer and S. Solimini, "On periodic solutions of nonlinear differential equations with singularities," Proceedings of the American Mathematical Society, vol. 99, no. 1, pp. 109-114, 1987.

[8] X. H. Tang and J. Jiang, "Existence and multiplicity of periodic solutions for a class of second-order Hamiltonian systems," Computers \& Mathematics with Applications, vol. 59, no. 12, pp. 3646-3655, 2010.

[9] Y. Wang, "Novel existence and uniqueness criteria for periodic solutions of a Duffing type p-Laplacian equation," Applied Mathematics Letters, vol. 23, no. 4, pp. 436-439, 2010.

[10] X. Yang, "Multiple periodic solutions for a class of second order differential equations," Applied Mathematics Letters, vol. 18, no. 1, pp. 91-99, 2005.

[11] A. Goppold, "Information and third order ontology," BioSystems, vol. 46, no. 1-2, pp. 169-173, 1998.

[12] J. Feigenbaum, "Second-, third-, and higher-order consumption functions: a precautionary tale," Journal of Economic Dynamics and Control, vol. 29, no. 8, pp. 1385-1425, 2005.

[13] P. G. Jones and P. K. Thornton, "Fitting a third-order Markov rainfall model to interpolated climate surfaces," Agricultural and Forest Meteorology, vol. 97, no. 3, pp. 213-231, 1999.

[14] S. Montant, E. Freysz, and M. Couzi, "Nuclear and electronic contributions to the third-order nonlinearity in different glasses," Optics Communications, vol. 281, no. 4, pp. 769-775, 2008.

[15] J. Chu and Z. Zhou, "Positive solutions for singular nonlinear third-order periodic boundary value problems," Nonlinear Analysis: Theory, Methods \& Applications, vol. 64, no. 7, pp. 1528-1542, 2006. 
[16] Z. J. Du, W. G. Ge, and M. R. Zhou, "Singular perturbations for third-order nonlinear multi-point boundary value problem," Journal of Differential Equations, vol. 218, no. 1, pp. 69-90, 2005.

[17] Y. Q. Feng, "Existence and uniqueness results for a third-order implicit differential equation," Computers \& Mathematics with Applications, vol. 56, no. 10, pp. 2507-2514, 2008.

[18] Y. X. Li, "Positive periodic solutions for fully third-order ordinary differential equations," Computers \& Mathematics with Applications, vol. 59, no. 11, pp. 3464-3471, 2010.

[19] S. Mukhigulashvili, "On a periodic boundary value problem for third order linear functional differential equations," Nonlinear Analysis: Theory, Methods \& Applications, vol. 66, no. 2, pp. 527535, 2007.

[20] R. P. Agarwal, Boundary Value Problems for Higher Order Differential Equations, World Scientific Publishing, River Edge, NJ, USA, 1986.

[21] D. R. Anderson, "Green's function for a third-order generalized right focal problem," Journal of Mathematical Analysis and Applications, vol. 288, no. 1, pp. 1-14, 2003.

[22] H. Brezis and J. Mawhin, "Periodic solutions of the forced relativistic pendulum," Differential and Integral Equations, vol. 23, no. 9-10, pp. 801-810, 2010.

[23] A. H. Nayfeh and D. Mook, Nonlinear Oscillations, Wiley, New York, NY, USA, 1979.

[24] J. Mawhin, "Global results for the forced pendulum equations," in Handbook on Differential Equations: Ordinary Differential Equations, A. Cañada, P. Drábek, and A. Fonda, Eds., vol. 1, pp. 533-589, Elsevier, Amsterdam, The Netherlands, 2000.

[25] R. Ortega, E. Serra, and M. Tarallo, "Non-continuation of the periodic oscillations of a forced pendulum in the presence of friction," Proceedings of the American Mathematical Society, vol. 128, no. 9, pp. 2659-2665, 2000.

[26] P. J. Torres, "Existence and stability of periodic solutions for second-order semilinear differential equations with a singular nonlinearity," Proceedings of the Royal Society of Edinburgh, Section A: Mathematics, vol. 137, no. 1, pp. 195-201, 2007.

[27] P. Tomiczek, "Variational approach to a periodic problem with friction," Nonlinear Analysis: Theory, Methods \& Applications, vol. 71, no. 12, pp. e1560-e1566, 2009.

[28] D. Guo and V. Lakshmikantham, Nonlinear Problem in Abstract Cones, Academic Press, New York, NY, USA, 1988. 


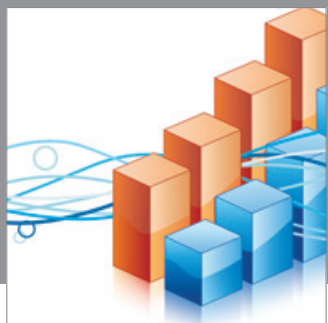

Advances in

Operations Research

mansans

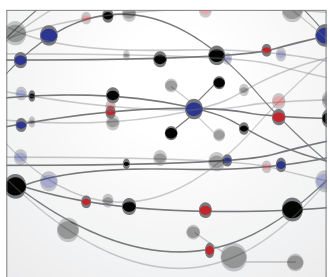

The Scientific World Journal
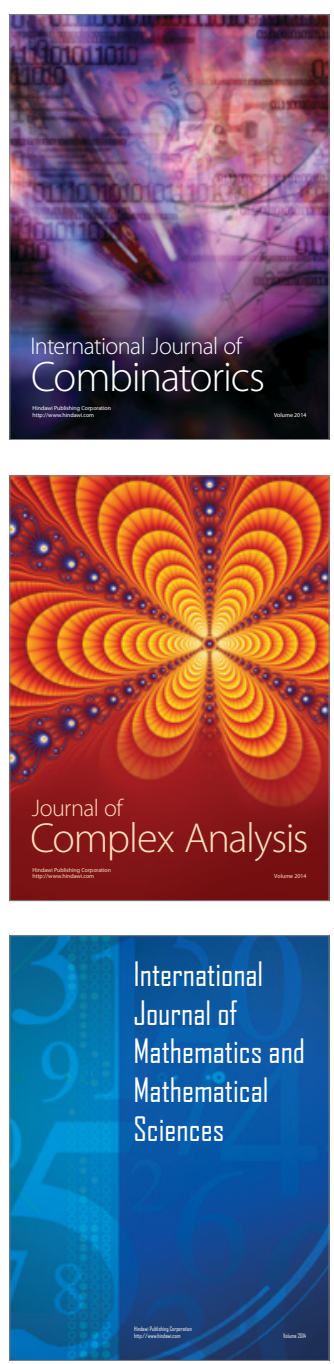
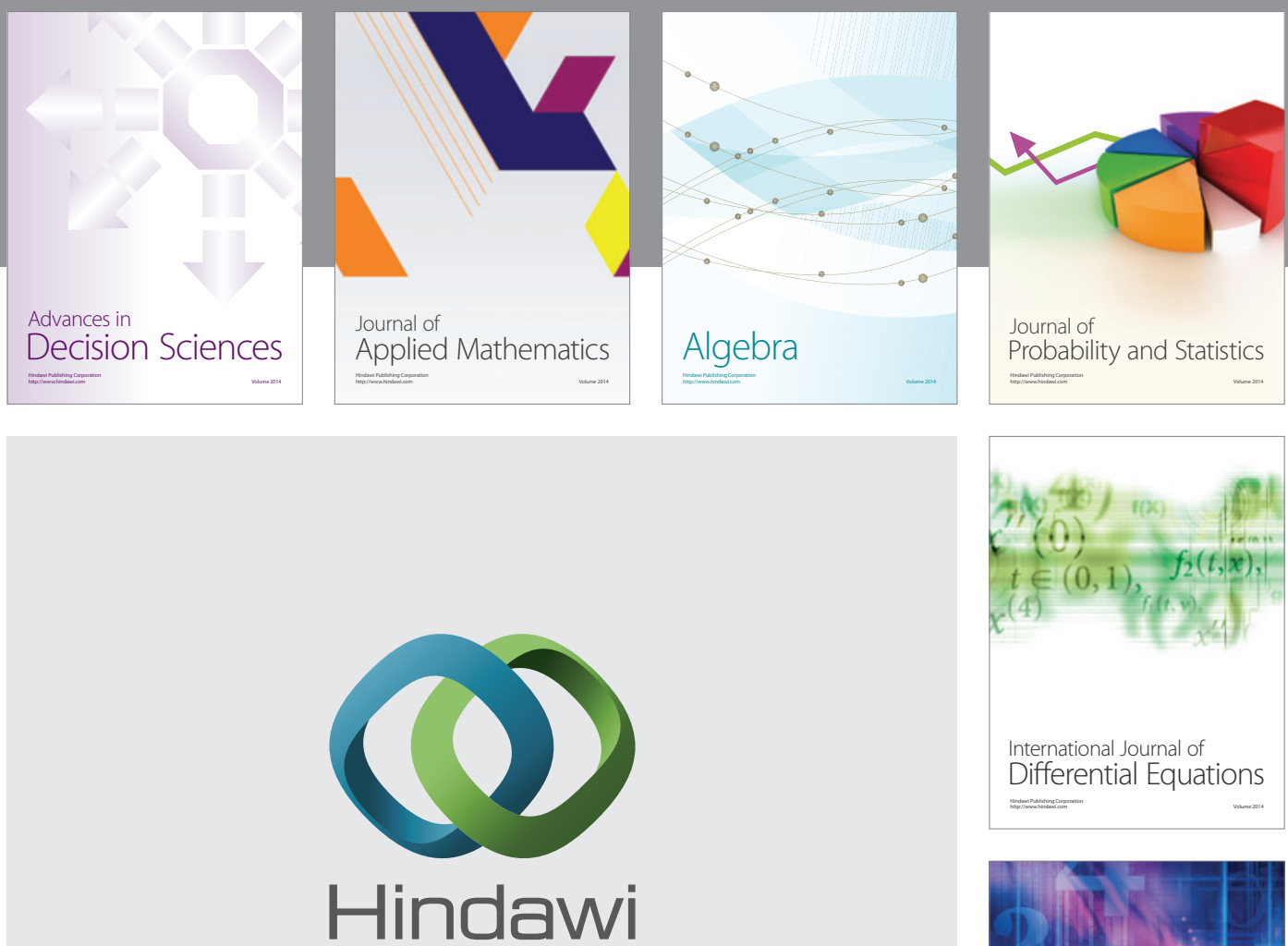

Submit your manuscripts at http://www.hindawi.com
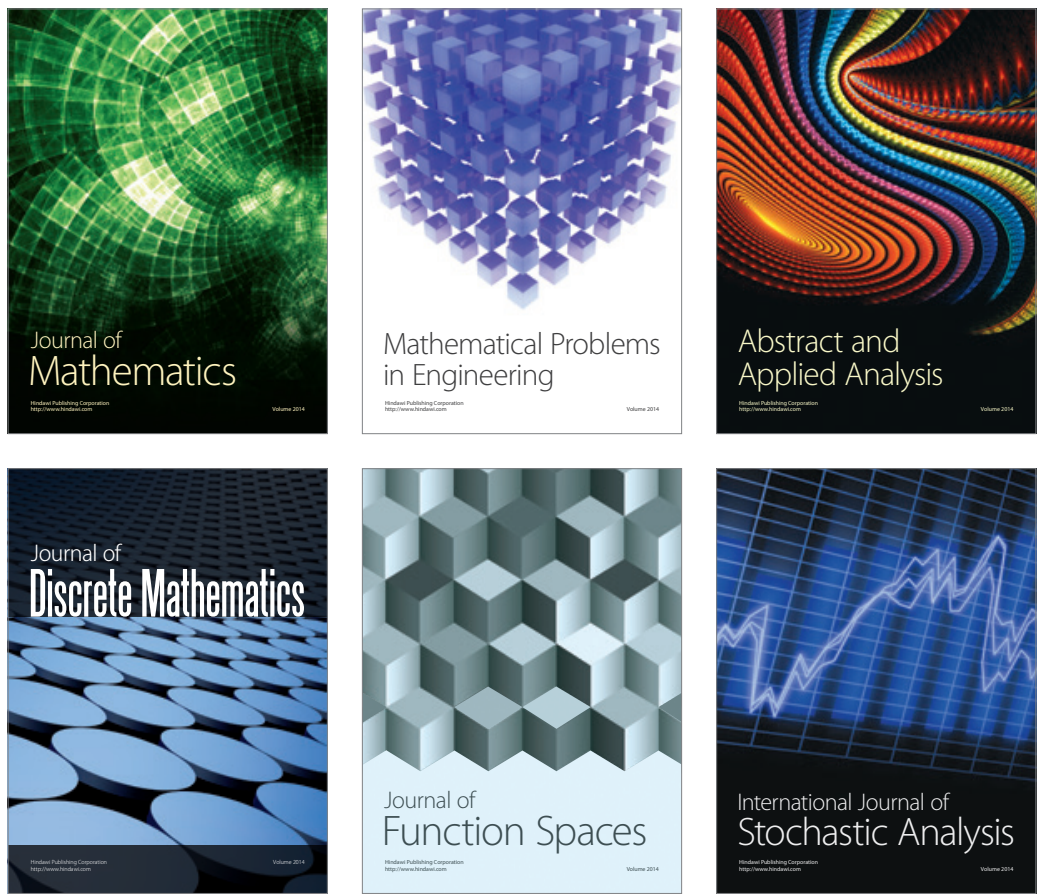

Journal of

Function Spaces

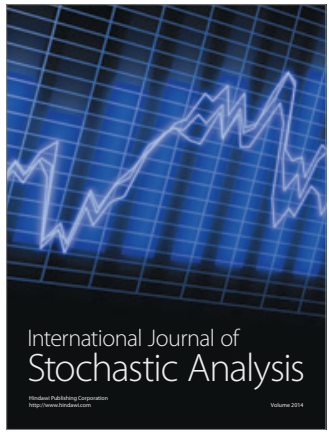

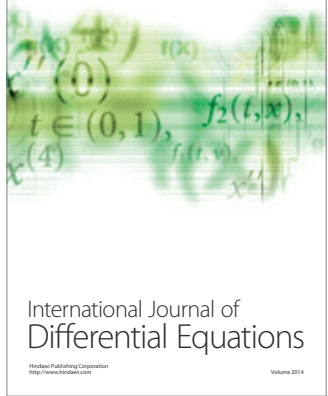
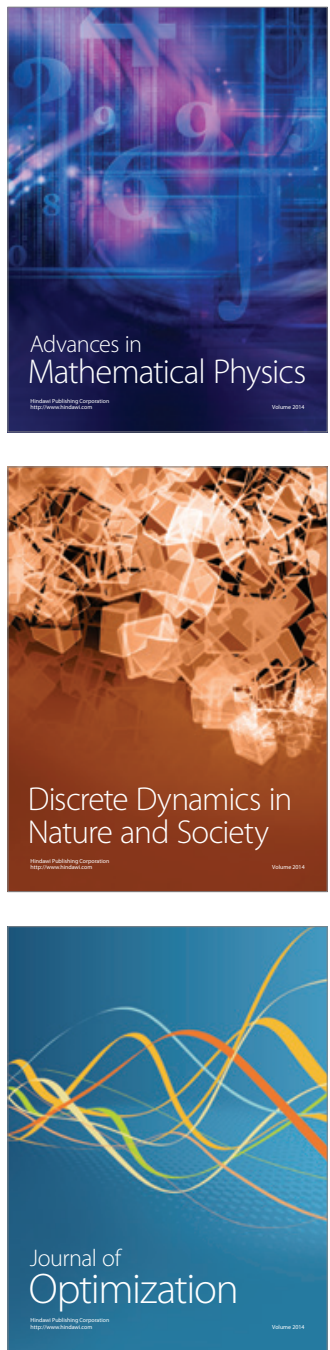